\title{
COMMUNICABLE DISEASES, NSW: NOVEMBER 2001
}

\section{TRENDS}

Notable features of communicable disease reports received by the NSW Department of Health through to September (Figure1, Table 2) include:

- the continuing pertussis epidemic that seems to be primarily affecting older children and adults and rural dwellers;

- the number of cases of meningococcal disease peaked in August (31 cases), with cases predominant among small children and young adults.

\section{INFLUENZA IN NSW, 2001}

\section{Rob Menzies, Elizabeth Anne Griggs, and Valerie Delpech}

The 2001 influenza season in NSW was a relatively mild one. Reports from general practitioners of 'influenza-likeillness' peaked in late July at 33 cases per 1,000 consultations, slightly lower than last year's peak of 37 . Routine laboratory diagnoses-predominantly from specimens from hospitalised children with respiratory illnesses-peaked in early August at 5.9 positives per 100 samples, the lowest rate reported in more than four years. Laboratory testing of specimens from patients of sentinel general practitioners (Directed Virological Surveillance) also peaked in early August with a predominance of influenza type A. The H1N1 A/New Caledonia/20/99-like strain, by far the most common (94 per cent of samples), was included in the Australian vaccine this year. For Australia and New Zealand as a whole, the A/New Caledonia/20/99 (H1N1) was predominant (77 per cent of isolates). Last year, by contrast, type A H3N2 predominated.

Many thanks to all who participated in the influenza surveillance-NSW public laboratories, public health units, many general practitioners, the Australian Sentinel Practice Research Network, and the World Health Organization Collaborating Centre for Influenza.

Weekly reports are coordinated by the NSW Department of Health between May and September and are available from the Communicable Disease Surveillance and Control Unit, NSW Department of Health, by contacting the Influenza Surveillance Officer on (02) 93919234.

\section{QUARTERLY REPORT: AUSTRALIAN CHILDHOOD IMMUNISATION REGISTER}

The table below reports immunisation coverage by area health service for children aged 12 months to less than 15 months.

These data refer to two different cohorts of children whose age has been calculated 90 days before data extraction. The information contained in each of the reports has been extracted from the Australian Childhood Immunisation Register (ACIR) and may not reflect actual coverage due to under-reporting. Fit

\section{TABLE 1}

IMMUNISATION COVERAGE BY AREA HEALTH SERVICE FOR CHILDREN AGED 12 MONTHS TO LESS THAN 15 MONTHS

\begin{tabular}{|lcc|}
\hline Area Health Service & 30 June 01 & 30 Sept 01 \\
\hline Central Coast & 94 & 93 \\
Central Sydney & 91 & 89 \\
Hunter & 94 & 96 \\
Illawarra & 92 & 93 \\
Northern Sydney & 90 & 89 \\
South Eastern Sydney & 89 & 89 \\
South Western Sydney & 92 & 90 \\
Wentworth & 92 & 92 \\
Western Sydney & 89 & 90 \\
Far West & 87 & 92 \\
Greater Murray & 93 & 93 \\
Macquarie & 93 & 92 \\
Mid North Coast & 91 & 91 \\
Mid Western & 90 & 92 \\
New England & 92 & 92 \\
Northern Rivers & 86 & 86 \\
Southern & 91 & 91 \\
\hline NSW & 91 & 91 \\
\hline
\end{tabular}


FIGURE 1

REPORTS OF SELECTED COMMUNICABLE DISEASES, NSW, JANUARY 1996 TO SEPTEMBER 2001, BY MONTH OF ONSET

These are preliminary data: case counts for recent months may increase because of reporting delays. Laboratory-confirmed cases, except for measles, meningococcal disease and pertussis.

cases

\begin{tabular}{|rc|}
\hline \multicolumn{2}{|c|}{ Jun-Sept 01} \\
Male & $54 \%$ \\
$<5$ & $0 \%$ \\
$5-24$ & $12 \%$ \\
$25-64$ & $73 \%$ \\
$65+$ & $14 \%$ \\
Rural & $89 \%$ \\
\hline
\end{tabular}

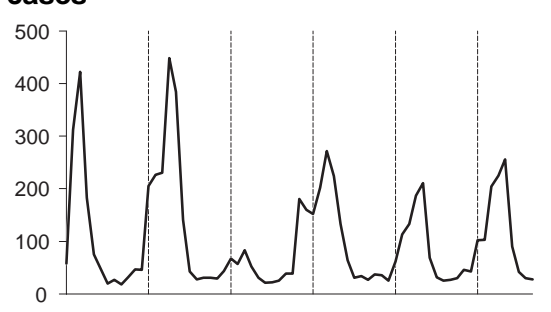

Arbovirus

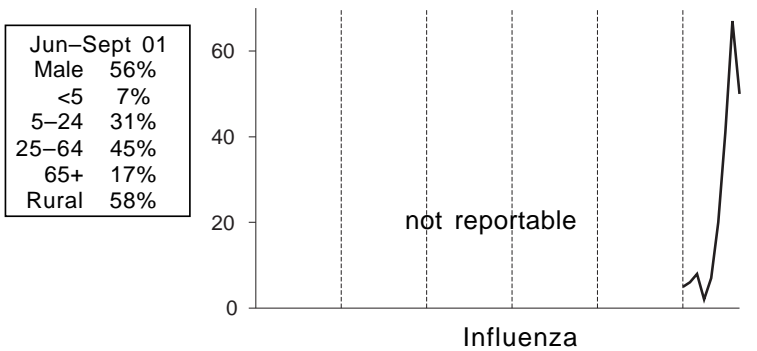

\begin{tabular}{|rc|}
\hline \multicolumn{2}{|c|}{ Jun-Sept 01} \\
Male & $90 \%$ \\
$<5$ & $2 \%$ \\
$5-24$ & $22 \%$ \\
$25-64$ & $77 \%$ \\
$65+$ & $0 \%$ \\
Rural & $13 \%$ \\
\hline
\end{tabular}

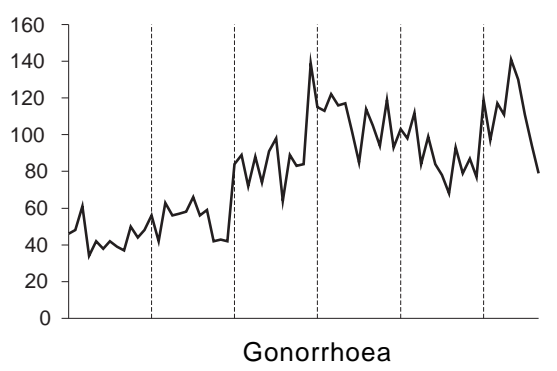

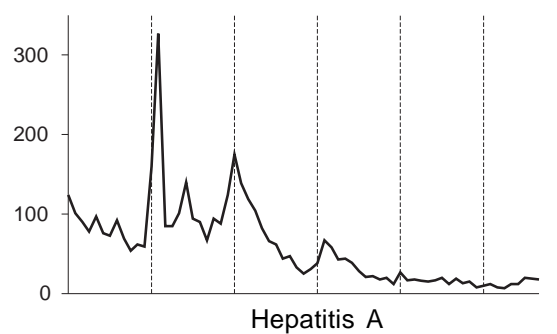
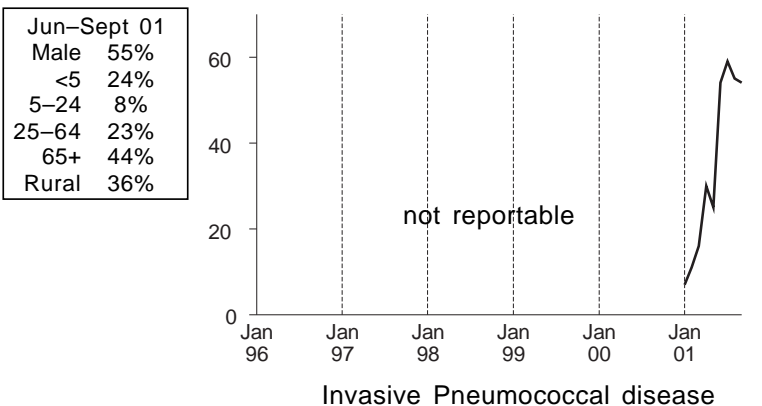

Invasive Pneumococcal disease
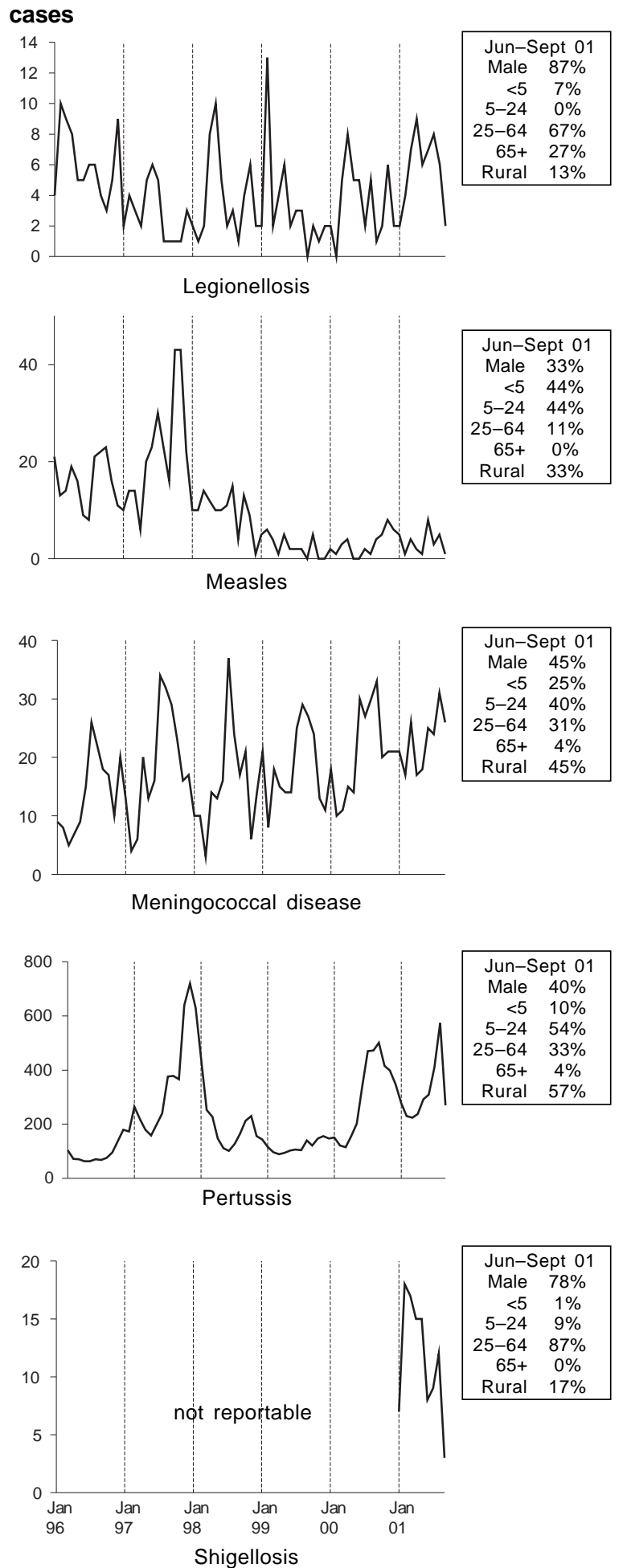

* For definition, see NSW Public Health Bulletin, April 2000 
and sexually transmitted

Gonorrhoe* (genital)*

Hepatitis B - acute vira

Hepatitis C - acute vira

HIV intis D - unspecified

Syphilis

Vector-borne

Arboviral infection $(\mathrm{BFV})^{*}$

Arboviral infection (Other)
Arboviral infection $(\mathrm{RRV})^{*}$

Arbovira
Malaria*

Zoonoses
Anthrax

Anthrax
Brucellosis

Leptospirosis

Lyssavirus

Psittacosis

Respiratory and other

Blood lead level

Invasive pneumococcal infection

Legionnaires' longbeachae*

Legionnaires' pneumophila

Legionnaires' (Other)

Leprosy

Meningococcal infection (invasive)

Vaccine-preventable

Adverse event after immunisation

H.influenzae $b$ infection (invasive)

Measles

Pertussis

Rubella*

Tetanus

Botulism

Cholera $^{*}$
Cryptosporidiosis

Food borne illness (not otherwise specified)

Gastroenteritis (in an institution)

Giardiasis* $^{*}$

Haemolytic uraemic syndrome

Hepatitis A

Hepatitis $E^{*}$

Listeriosis"

Shigellosis

Typhoid and paratyphoid*

Verotoxin producing $\mathrm{E}$. coli

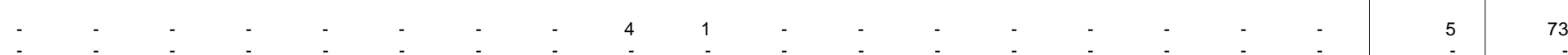

* lab-confirmed cases only

$\begin{array}{rrrrrrrrr}41 & 41 & 28 & 10 & - & 2 & 27 & 12 & 78 \\ 22 & 5 & 5 & 1 & 1 & - & 1 & 1 & 41 \\ - & 1 & - & - & - & - & - & - & \\ 41 & 45 & 66 & 6 & - & 2 & 11 & 9 & \\ 1 & - & 1 & & - & -28 & 56 & 22 & 82\end{array}$

CSA $=$ Central Sydney Area

$W S A=$ Western Sydney Area 